\title{
Design and Application of Residential Area Noise Monitoring Based on Wireless Sensor
}

\author{
https://doi.org/10.3991/ijoe.v13i07.7276 \\ Qiuling Zheng \\ Jilin Jianzhu University, Jilin, China \\ $12674726 @$ qq. com
}

\begin{abstract}
A new node deployment optimization algorithm is proposed to solve deployment problems of the monitoring terminal. Based on wireless sensor, a kind of deployment optimization algorithm of sensor nodes location is put forward, and OMNET++ simulation is used for verification. The experimental results show that this deploy method can get good applications in different areas of the city and achieve higher deployment results. Therefore, it is concluded that the proposed algorithm is feasible and effective.
\end{abstract}

Keywords - wireless sensor, nodes deploy, OMNET++

\section{Introduction}

Noise pollution has become a worldwide problem at present, which directly interferes with the normal life, work and study, and seriously affects people's health. As a result, people have established a variety of noise pollution monitoring systems to try to grasp the detailed situations of noise pollution, and try to control or reduce the noise pollution. Most of the traditional noise monitoring systems adopt wired network connection, which is composed of the specified monitoring points deployment technologies. This kind of system deployment and maintenance costs are high, and it is difficult to transmit the data to the user in real time, effectively and reliably [1]. The noise monitoring system based on the wireless sensor network can effectively improve the detection quality, and reduce deployment and maintenance costs. The noise monitoring system has the advantages of a wide range of monitoring, able to achieve all-day and real-time monitoring, and it supports a variety of access methods and so on.

The coverage quality of sensor networks depends largely on the deployment method of sensor networks, and the deployment mode also affects the performance of sensor networks, like survival time, connectivity and so on. As a result, researchers at home and abroad have a lot of researches on the deployment mode of sensor nodes. In the field of noise monitoring based on wireless sensor, the researchers also studied the deployment problem of noise monitoring points, and put forward the optimization scheme. However, people always want to solve this problem, but the solution is still not perfect and the optimization effect is not very ideal. In the paper, the node de- 
ployment optimization problem in allusion to the noise network is studied, a monitoring point deployment optimization strategy and method is put forward, and simulation software OMNET++ is used for simulation analysis, to illustrate the feasibility and effectiveness of the algorithm.

\section{State of the Art}

Node deployment is the arrangement of nodes, to arrange in a reasonable manner, within the specified range of detection, so as to meet the needs of monitoring department for monitoring. The first step of noise monitoring network is to deploy the nodes in the network. The standard node deployment can not only improve the efficiency of network operation and better make use of network resources, but also change the number and activity degree of nodes according to different monitoring requirements. Reasonable adjustment of the density of network nodes can get more complete and accurate network monitoring information [2].

In foreign countries, the problem of node deployment optimization has been paid more attention to, and it is the most basic element for the realization of the application of noise monitoring network. Singh pointed out that the number and location of nodes of different deployment methods are also different, and the purpose is to establish an efficient detection system with a powerful node utilization function. What plays the decisive role in the practicability and effectiveness of the network is the node coverage quality, node deployment, network connectivity and lifetime [5]. In addition, the limited energy supply and limited sensing radius will limit the processing capacity and resources of the monitoring node [3]. Faced with this situation, the challenges of limited noise monitoring node resource device and the establishment of long duration and high reliability applications requirements, designers need to constantly improve the node position accuracy and high utilization rate. Only in this way can it solve the influence of its own characteristics.

In the preparation stage of the deployment, we can calculate the number of nodes needed in the area to be detected according to this function, and can adjust the distance of communication between the nodes and calculate the area range. In the monitoring system of the mobile node, we can adjust the deployment of nodes through moving the node, optimize the distribution of nodes, enhance the ability of communication between the nodes, and detect the largest area with the least nodes. The virtual force algorithm can be used to calculate the node's moving trajectory, and to solve the problem of the node's location. [6].

The deployment can be divided into two ways, namely, static and dynamic deployment these two kinds. Static deployment is the to determine the distribution of nodes by using the optimal method based on a full understanding of the environment, usually fixed on the placement before the system starts, and generally the location of nodes will not change [4]. For instance, the airport noise monitoring system implemented by Italy, the United States and other countries is to make use of static deployment mode. Dynamic deployment is to adjust according to the changes of the 
network environment, so the dynamic deployment must monitor the network, and the node will change the orientation according to the change.

\section{$3 \quad$ Methodology}

In the noise monitoring system, because the distribution of noise signal is not continuous, and it will be affected by the external environment, it is particularly important to design a reasonable stationing. First of all, we discuss the problems of noise monitoring distribution:

The stationing problem of sensitive area in noise monitoring:

According to the stipulation of noise monitoring standard, we should place the noise monitoring point in the sensitive area outside of 1 meter. However, in the practical process, we will encounter many problems. For example, if the noise source is closed or covered, then the noise will be reduced. At the same time, if we cannot measure noise synchronously, then it is impossible to get the results that can reflect the real monitoring data in the area. In this standard, there is no relationship between the internal noise source and the sensitive building. But in practice, we will often encounter such a situation.

The problem of stationing time in noise monitoring:

According to the stipulation of noise monitoring standard, in the normal working day and measuring time, we commonly use short-term sampling measurement. But there is not a specific provision about the measuring time, so the time can be half a minute, 10 minutes or longer. The standard specifies that the measuring time of steady-state noise is 1 minute. For periodic noise, the point measurement time is the whole cycle, where it is relatively easy to measure the steady-state noise distribution. However, for non-steady-state noise of periodic and non-periodic, it is difficult to control the time in the point of its measurement.

Based on these questions, we have made the following three countermeasures:

Countermeasure of stationing time problem in noise monitoring:

For periodic noise measurements, each point is 10 minutes or 20 minutes. And for noise measurements of non-periodic non-steady-state, each point is 1 hour or 24 hours for fixed-point measurements. For noise measurements in urban environments, the time can be specified as 5 minutes, and measure with the AWA6218B type noise analyzer. For the measurement of construction site, it should be in the case of continuous construction site, we should carry more than one instrument to monitor when the construction works in the normal construction period.

Countermeasure for reasonable selection of stationing in noise monitoring:

In noise monitoring system, we should lay out point in many places and confirm the location from the sensitive place which we have find. When the standard stationing position differs from the sensitive position, we should monitor the location of the standard stationing and sensitive stationing concurrently to meet the actual monitoring situation. When the stationing meets the requirements of the standard, but sensitive buildings have produced the actual noise, this time the sensitive buildings should be taken as a stationing instead of laying out point with the standard. In noise monitoring 
system, the strategy of stationing background usually has the following three methods related to the stationing strategy in the background value. Firstly, the test time should be selected in a relatively quiet time period, when under the environment with high background value, and the sound level difference is less than $3 \mathrm{~dB}$, it is necessary to re-measure it. We can arrange the measurement in a quiet environment like putting the measurement time in the evening. Secondly, we need to test it using the method of startup-shutdown. This method is more suitable for steady-state noise measurement. The third step is the choice of stationing, when the measured sound source cannot stop working and cannot obtain the background value, we can change the location according to the situation. The location is equal to the background value here. For example, if an arterial traffic adjoins a building site, we can measure the traffic noise near the traffic trunk at the construction site. For the non-steady-state noise of nonperiodic, the background noise is relatively low and stable. We can take the L90 value at a period of time as the background value according to the situation. We need to exclude some occasional factors in measuring the background value. Usually, we correct the strategy taken as background value according to the calculation method, and the formula is: $L_{2}=10 \operatorname{Lg}\left(10 L_{0} / 10-10 L_{1} / 10\right)$. Among this, Lo is the measured value of noise, L1 is the value of background noise, and L2 is the contribution value or the correction value of noise [9].

The defects of node deployment research and limitations not fully considered by the previous academic research circle are shown as the following three main points:

Communication model and perception lack practicality. At present, most of the algorithms assume that the sensor nodes are too idealistic, and how to establish a realistic perception model is a problem to be solved.

Deployment differences cannot be ignored. The deployment problem of sensor is established in the case that all the regions have the same detection demand, while for the noise monitoring system, different regions have quite different requirements for the density requirements of monitoring points. For example, monitoring of the living area is stricter than the noise monitoring of factory areas [6].

Ignore the boundary factors effects. Do not take into account the impact of the monitoring area boundaries. In the sensor model, the boundary problem is not well considered.

In order to solve the above problems, we first of all propose the optimization goal of the system node deployment:

Coverage and connectivity: for wireless sensor networks, the network coverage is the most important, which is the basis for us to complete the tasks of monitoring. For different regions, first of all, we need to cover it by the sensor network, and on the basis of this, other applications can be carried out smoothly.

The minimum nature of deployment resources. Under the condition of meeting the coverage requirements, we propose that the deployed nodes need to be as small as possible, so as to save node resources.

The lifetime of the sensor is supposed to be as long as possible: it is necessary to make requirements for the continuity of the node deployment problem, so in the future, the energy balance algorithm for the sensor can be well implemented, to extend the life of the sensor network. 
The performance of deployment optimization algorithm: the deployment of sensor nodes needs to consider the time and space performance, and get the scheme how to deploy a fixed area as soon as possible.

Other related function support: the target of perception information and monitoring environment of the system, but the perceived final target needs to make transmission and processing of the perceived information, then the deployment methods need to take into account the support of the follow-up data for processing method, so the follow-up data transmission and processing can be more convenient.

According to the optimization target mentioned above, this section proposes a node deployment method as far as possible to meet the optimization objectives. The method can satisfy that the system may complete the regional coverage in a limited area with less nodes. At the same time, the deployment of nodes does not consider the block characteristics of the region, and in noise monitoring environment, the requirements of different areas for monitoring terminal lead to different distribution density.

First of all, we introduce several definitions:

Define 1: the monitoring area set $\mathrm{R}=\{\mathrm{R} 1, \mathrm{R} 2, \ldots, \mathrm{Rp}\}$. The region set is defined as the monitoring range. Different regions have different coverage requirements weights for indicating the importance of the region. We consider the monitoring area of three $\mathrm{Lp} * \mathrm{Hp}$ rectangular monitoring areas.

In the application of the noise monitoring system, we assume that the monitoring area is a regular rectangle. In the rectangular region, we study the deployment mode of nodes.

Define 2: expected deployment quality. For a sensor network and the area to be deployed, the coverage area that the deployed sensor nodes reaches is the actual area, and there is also an area that the user is expected to cover. As a result, the expected deployment quality is defined, that is, in the deployment process of a sensor network, the percentage of the coverage area of the deployed nodes in the whole monitoring area [7]. This ratio is called the expected deployment quality. At the same time, the overall expected deployment quality is equal to the weight of all the individual regions. The formula is shown in (1).

$$
\sum_{k=1}^{p} \lambda_{k} \frac{\left\|\left(\bigcup_{i=1}^{n_{k}} S\left(r_{i}\right) \cap R_{k}\right)\right\|}{\left\|R_{k}\right\|}
$$

In (1), $S\left(R_{I}\right)$ represents the perception area of the node $i, n$ refers to the amount of node in the monitoring area, and $\|\mathrm{R}\|$ indicates the area of monitoring region. Each region is similarly assumed as a rectangular, the length and height are $\mathrm{L}_{\mathrm{k}}$ and $\mathrm{Hk}$, respectively.

The given monitoring region is $\mathrm{R}=\left\{\mathrm{R}_{1}, \mathrm{R}_{2}, \ldots, \mathrm{R}_{\mathrm{p}}\right\}$, and the perception area of node is $\mathrm{S}\left(\mathrm{r}_{\mathrm{i}}\right)$. It is necessary to consider how to deploy the sensor, so in the case of fixed sensor amount of $\mathrm{n}$, to get the maximum expected deployment quality.

Making use of mathematical model description, as shown in (2): 


$$
\begin{array}{r}
\operatorname{Max} \sum_{k=1}^{p} \lambda_{k} \frac{\left\|\left(\bigcup_{i=1}^{n_{k}} S\left(r_{i}\right) \cap R_{k}\right)\right\|}{\left\|R_{k}\right\|} \\
r_{1}=r_{2}=\ldots=r_{n} \\
\text { Subject to }\left\{\begin{array}{l}
\sum_{k=1}^{p} \lambda_{k}=1 \\
0<S\left(r_{i}\right) \leq \pi r_{i}^{2}
\end{array}\right.
\end{array}
$$

This paper puts forward a node deployment mode bade on average area coverage, to solve the problem of the maximum expected deployment quality.

The variable $\mathrm{V}$ is used to represent the area that cannot be covered by nodes in the monitoring area, $\mathrm{V}=\left\{\mathrm{V}_{1}, \mathrm{~V}_{2}, \ldots, \mathrm{V}_{\mathrm{p}}\right\}$, and for an area $\mathrm{V}_{\mathrm{k}}$ in it, we can obtain:

$$
V_{k}=\int_{R_{k}}(x) d x
$$

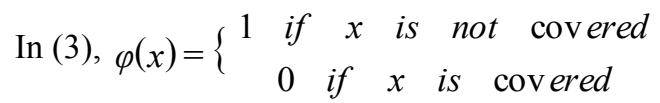

Then, the expected not to be covered area of a single area is:

$$
E\left(V_{k}\right)=\int_{R_{k}} E(\varphi(x)) d x
$$

The expected not to be covered area of the whole area is:

$$
E(V)=\sum_{k=1}^{p}\left(V_{k}\right)=\sum_{k=1}^{p} \int_{R_{k}} E(\varphi(x)) d x
$$

For a given arbitrary point $x \in R_{k}$, use $\beta(x)$ to represent the circular area with the center of $x$ and the area of $r, \beta(x)=\pi r^{2}$. Then, we can know that, if $x$ is covered, there is a sensor node within $\beta(x)$.

We define the sign $Q_{x i}$ as an arbitrary point $i \in R_{k}$ and the probability for not be covered, and take the point $\mathrm{X}$ selected for the monitoring area $\mathrm{R}$ as an event, then there will be $\mathrm{p}$ independent events. As a result, the probability for the point $\mathrm{X}$ not be covered by the randomly deployed point $i$ is:

$$
Q_{x i}=1-\sum_{k=1}^{p} \int_{R_{k}} \frac{E_{r}[k]}{\left\|R_{k}\right\|}
$$


In (6), $\mathrm{E}_{\mathrm{r}}[\mathrm{k}]$ is the average cover area of an arbitrary node in the area of $\mathrm{R}_{\mathrm{k}}$ in the point $\mathrm{x}$. Since that we assume that the probe radius of each probe is the same, then $\mathrm{E}_{\mathrm{r}}[\mathrm{k}]=\pi \mathrm{r}^{2}$, and (6) can be equivalent to:

$$
Q_{x i}=1-\sum_{k=1}^{p} \int_{R_{k}} \frac{\pi r^{2}}{\left\|R_{k}\right\|}=1-\pi r^{2} \sum_{k=1}^{p} \int_{R_{k}} \frac{1}{\left\|R_{k}\right\|}=1-\pi r^{2} \sum_{k=1}^{p} \int_{R_{k}} \frac{1}{L_{k} H_{k}}
$$

As a result, when there are $\mathrm{n}$ nodes deployed in the monitoring area $\mathrm{R}$, there will be:

$$
E(\varphi(x))=\prod_{i=1}^{n} Q_{x i}=\left(Q_{x i}\right)^{n}
$$

Based on the above equations, we can get the solution by using the solving method of exponential equation. Since that the solving process and analytical expression are very complex, it will not be described here. According to the specific numerical problems, we can quite easily find out the specific numerical equations by taking it into the solution.

\section{$4 \quad$ Result Analysis and Discussion}

We make use of the OMNET++ simulation software to simulate the deployment of nodes, the simulation background uses the noise monitoring deployment case of the map of Beijing City, where host refers to the monitoring points, with a total of 100 monitoring equipment, and server indicates the service station, containing the data management center and data processing center. We compare the difference by using the random deployment and the optimization deployment based on weight relevance. Because each region has a different focus of control, for instance, noise monitoring equipment in the vicinity of the city is more significant in the importance of monitoring the outskirts, it is necessary to arrange more monitoring points in the vicinity of the area, so as to reduce the noise monitoring blind zone and improve the noise monitoring accuracy. We divide the region into three parts, what is within the first ring is the region S1, what is out of the first ring and within the third ring is the region S2, and what is out of the third ring is S3, and the distribution of their importance is: 0.6, $0.3,0.1$. We study the difference between the random deployment quality and the deployment quality by using the weight-based solution. 


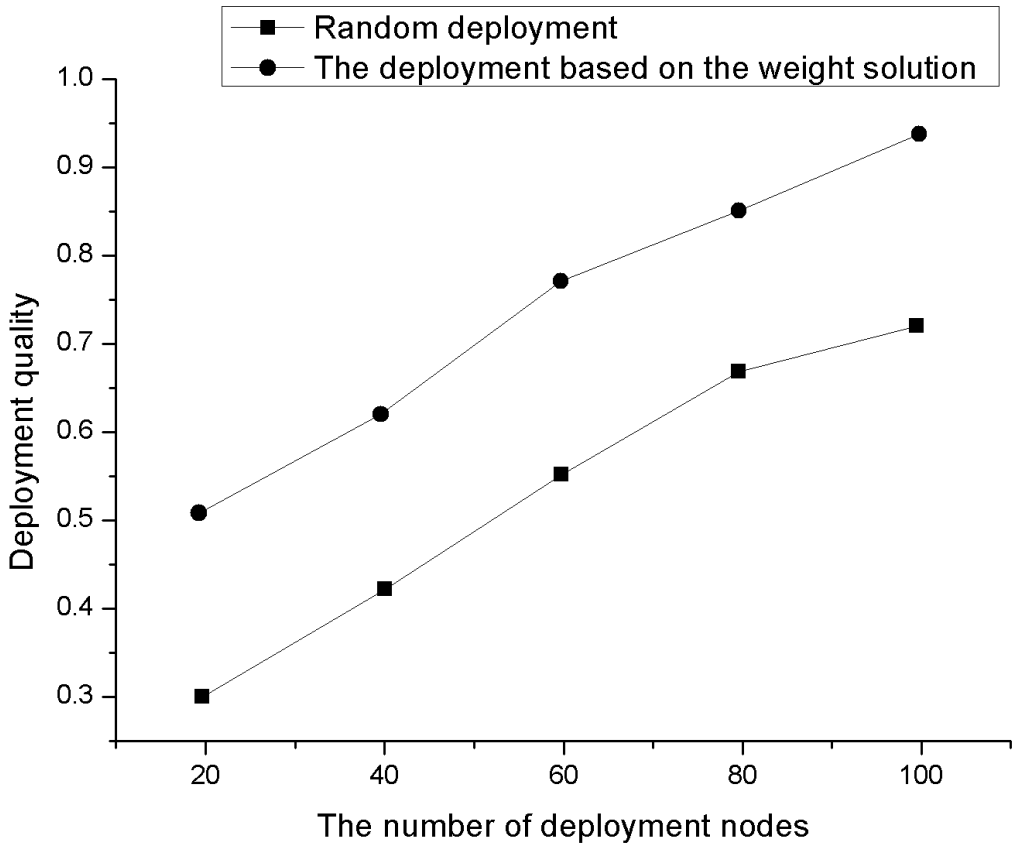

Fig. 1. Comparison between the random deployment and the deployment quality by using the weight-based solution

For the deployment of each area, we make use of fixed 100 noise monitoring and acquisition equipment, and analyze the impact of different weights on the number of deployments.

We assume that the weights of the three regions have the following 5 sets of data:

First group: $(0.5,0.3,0.2)$;

Second group: $(0.6,0.3,0.1)$;

Third group: $(0.7,0.2,0.1)$;

Fourth group: $(0.8,0.15,0.05)$;

Fifth group: $(0.9,0.075,0.025)$;

We compare the number of distributions and the number of random distributions obtained by the above equation respectively: 


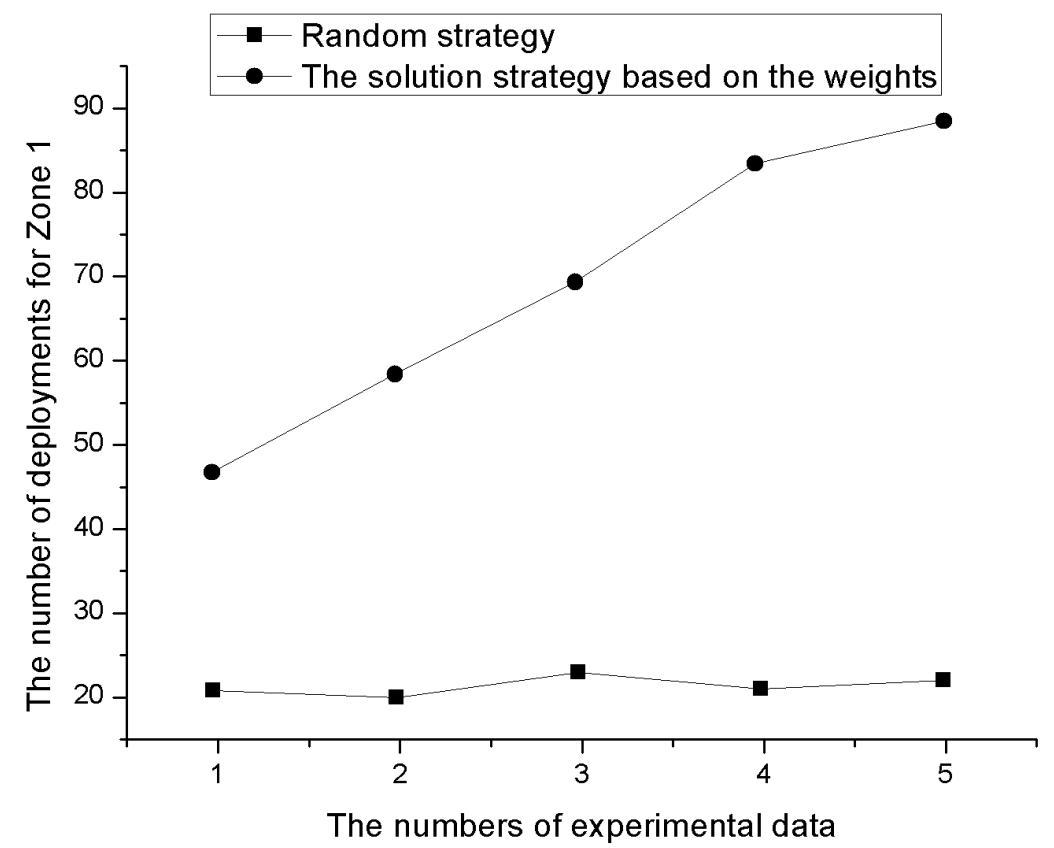

Fig. 2. The deployment number distribution of region 1

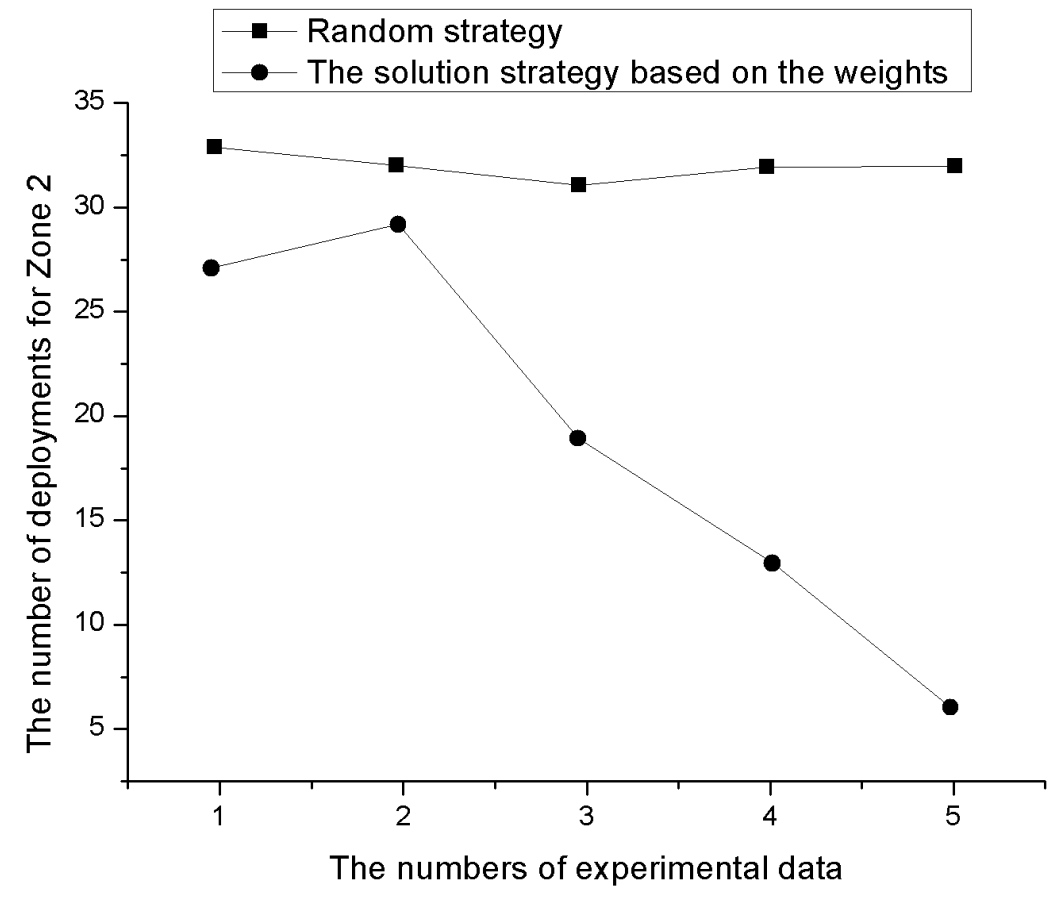

Fig. 3. The deployment number distribution of region 2 


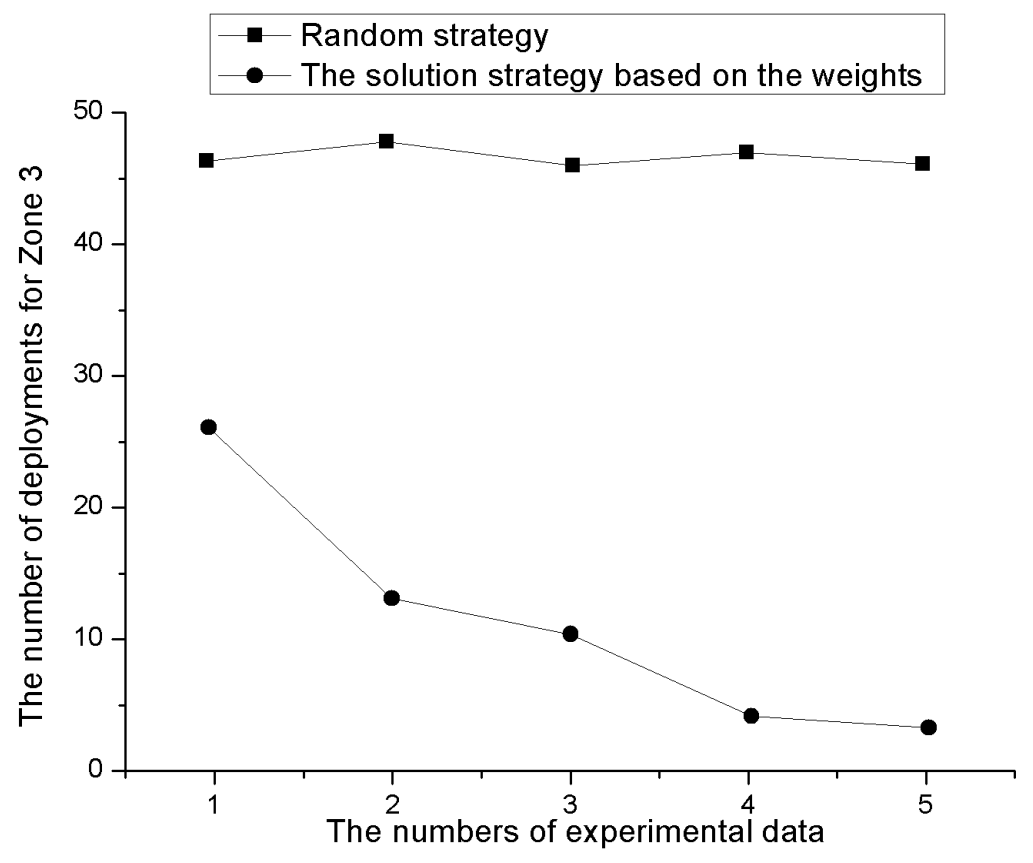

Fig. 4. The deployment number distribution of region 3

From the deployment distribution of different regions, it can be seen that, using the random deployment mode makes the deployment number of each region relatively fixed, unable to distinguish the importance of each unique area, which is obviously not conducive to the application of actual noise monitoring. Whereas, using our proposed monitoring node deployment based on the weight of the region can well distinguish the deployment mode of different regional monitoring nodes, so the key areas can have better monitoring capability.

\section{Conclusion}

The node deployment optimization problem of noise network is studied, and a monitoring point deployment optimization strategy and method is proposed. What's more, a simulation analysis is made by using the simulation software OMNET++. After analyzing the simulation results, it is concluded that the proposed monitoring node deployment based on the weight of the region can well distinguish the deployment mode of different regional monitoring nodes, so that the key areas can have better monitoring capability. Therefore, the proposed method is feasible and effective. However, there are still some shortcomings for the presented algorithm. Because the project development cycle is short, the hardware condition is limited and other factors, the system is not perfect, so it is possible to add more functions in the system. Therefore, the future environmental noise monitoring system will be more automated, integrated, and real-time. 


\section{$6 \quad$ References}

[1] Abdrabou, A. (2016). A wireless communication architecture for smart grid distribution networks. IEEE Systems Journal, 10(1): 251-261. https://doi.org/10.1109/JSYST.2014.230 $\underline{4291}$

[2] Bell, M. C., \& Galatioto, F. (2013). Novel wireless pervasive sensor network to improve the understanding of noise in street canyons. Applied Acoustics, 74(1): 169-180. https://doi.org/10.1016/j.apacoust.2012.07.007

[3] Cetinkaya, O., \& Akan, O. B. (2016). Use of Wireless Sensor Networks in Smart Homes. In Emerging Communication Technologies Based on Wireless Sensor Networks: Current Research and Future Applications (pp. 233-258). CRC Press. https://doi.org/10.1201/b200 $\underline{85-13}$

[4] Das, B. B., \& Ram, S. K. (2016, August). Localization using beacon in wireless sensor networks to detect faulty nodes and accuracy improvement through DV-Hop algorithm. In Inventive Computation Technologies (ICICT), International Conference on (Vol. 1, pp. 15). IEEE.

[5] Han, J., Lee, I., \& Kim, S. H. (2015). User-friendly monitoring system for residential PV system based on low-cost power line communication. IEEE Transactions on Consumer Electronics, 61(2): 175-180. https://doi.org/10.1109/TCE.2015.7150571

[6] Peng, C., Qian, K., \& Wang, C. (2015). Design and application of a VOC-monitoring system based on a ZigBee wireless sensor network. IEEE Sensors Journal, 15(4): 2255-2268. https://doi.org/10.1109/JSEN.2014.2374156

[7] Wessels, P. W., \& Basten, T. G. (2016). Design aspects of acoustic sensor networks for environmental noise monitoring. Applied Acoustics, 110: 227-234. https://doi.org/10.1016/j.apacoust.2016.03.029

[8] Tahouri S, Atani R E, Karbasi A H, et al. (2015). Application of connected dominating sets in wildfire detection based on wireless sensor networks, 3(2):139.

[9] Sagar E, Goel R. (2014). A New Approach to System Design for Disaster Management and Monitoring using Wireless Sensor Network. International Journal of Engineering Sciences \& Research Technology, 3(7).

[10] Radha V, Kaviyapriya P. (2014). CLUSTERING BASED CRITICAL EVENT MONITORING IN WIRELESS SENSOR NETWORKS. International Journal of Research in Computer Applications \& Robotics, 2(4).

\section{$7 \quad$ Author}

Qiuling Zheng is ith Jilin Jianzhu University, Jilin, China (12674726@qq.com). 